\title{
Correction to: Why lower educated people are more likely to cast their vote for radical right parties: Testing alternative explanations in The Netherlands
}

\author{
Michael Savelkoul $^{1} \cdot$ Peer Scheepers $^{1}$
}

Published online: 3 January 2019

(C) Springer Nature Limited 2019

\section{Correction to: Acta Politica (2017) 52, 544-573 \\ https://doi.org/10.1057/s41269-016-0031-z}

In the original publication of the article, Table 2 (Model 6) and Tables A1, A2, A3 in Appendix section contained some minor incorrectnesses. This has no impact on our conclusions, which remain unchanged. The correct Tables 2, A1, A2 and A3 are given below:

The original article can be found online at https://doi.org/10.1057/s41269-016-0031-z.

Michael Savelkoul

m.savelkoul@maw.ru.nl

1 Department of Sociology, Radboud University Nijmegen, P.O. Box 9104, 6500 HE Nijmegen, The Netherlands 


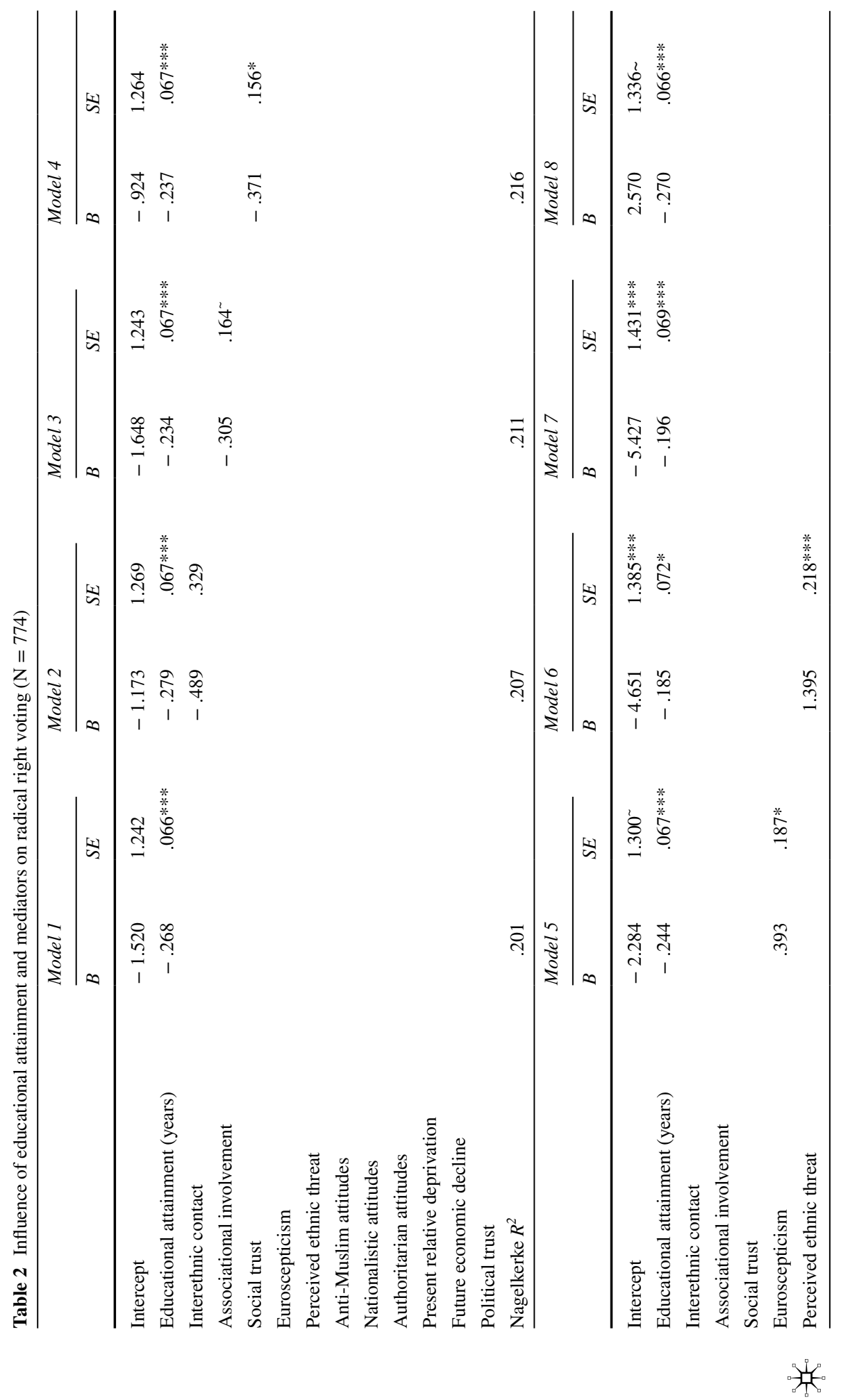




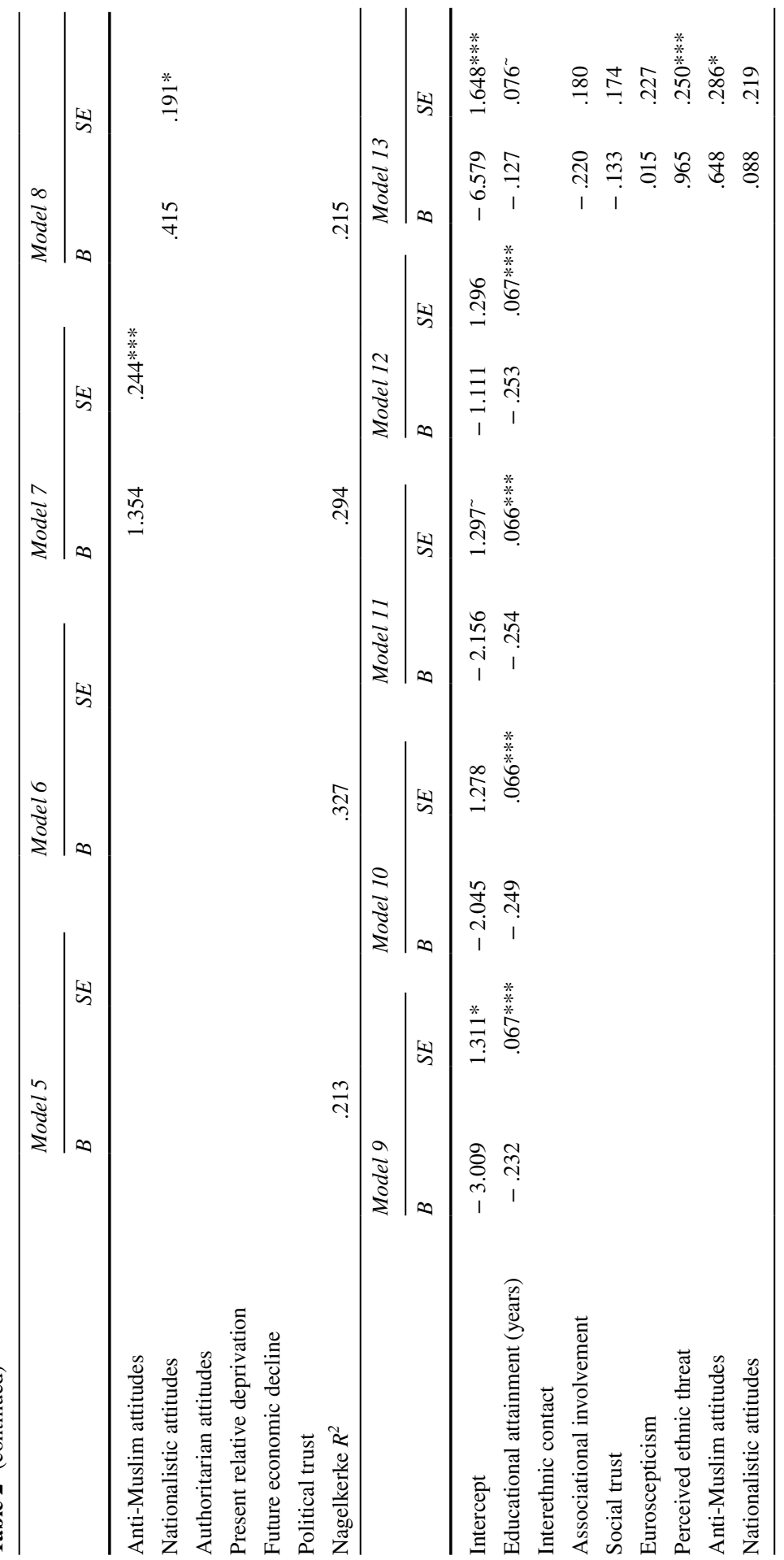

站。 


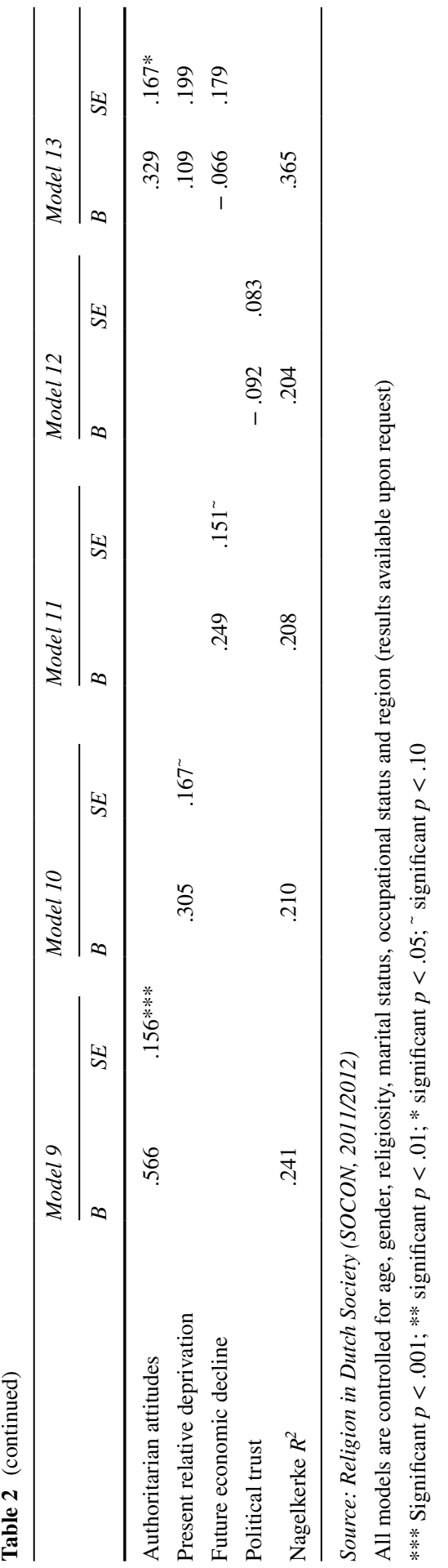




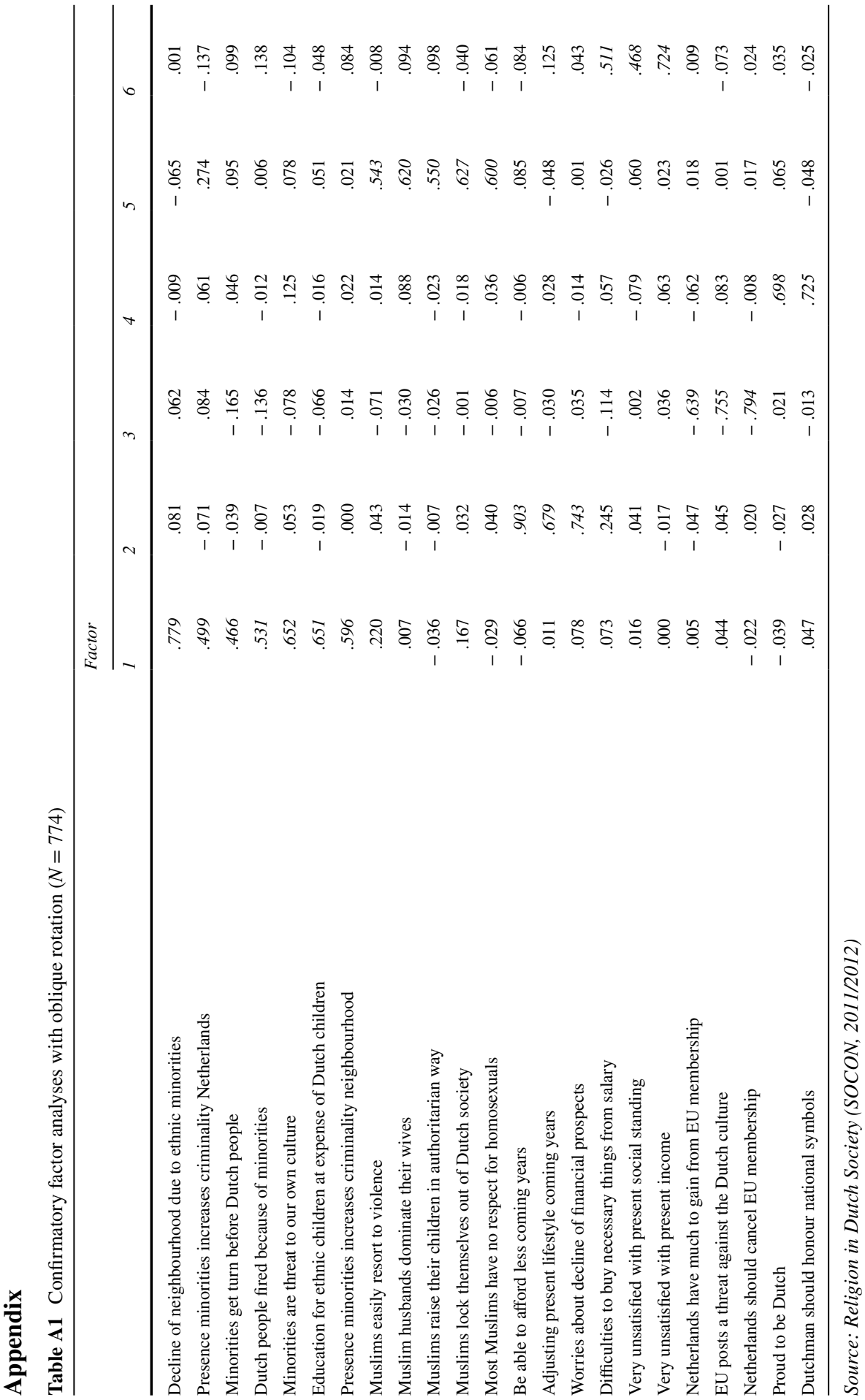

站的 


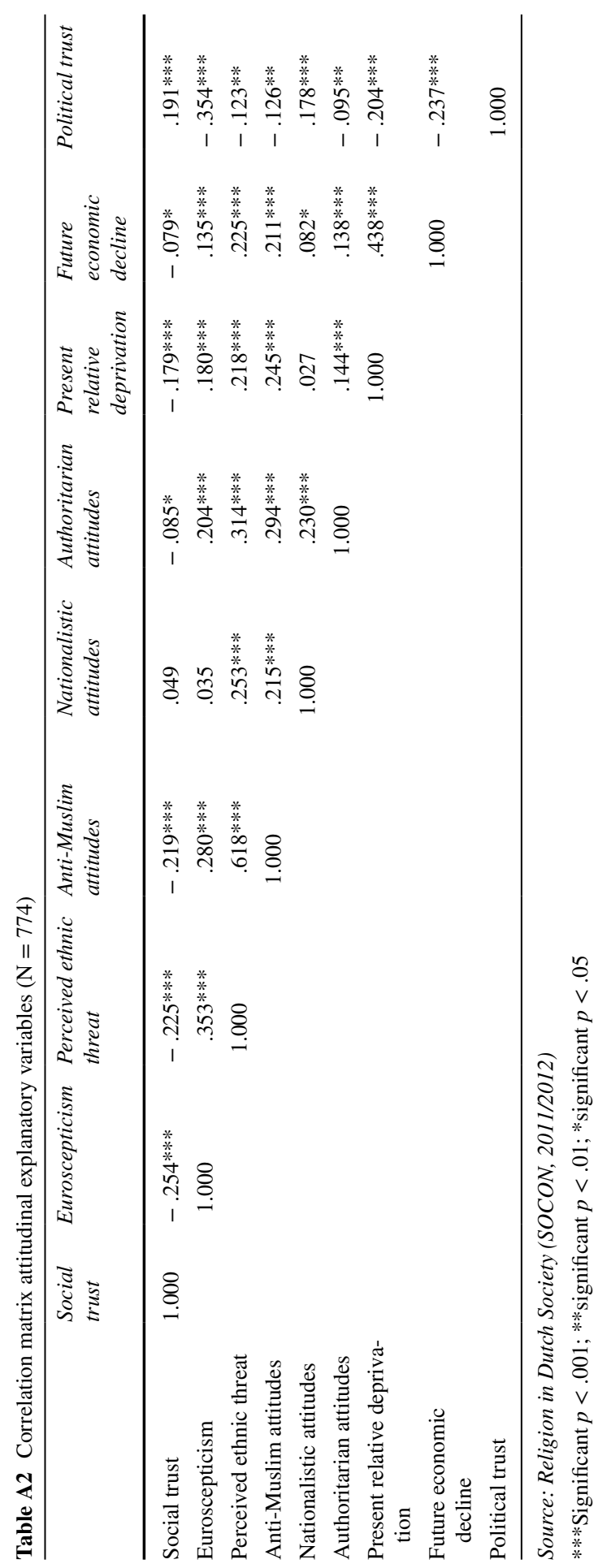


Table A3 Descriptive statistics $(\mathrm{N}=774)$

\begin{tabular}{|c|c|c|c|}
\hline & Range & Mean/per cent & Std. Dev. \\
\hline \multicolumn{4}{|l|}{ Dependent variable } \\
\hline Radical right voting (voting for the PVV) & $0 / 1$ & 8.40 per cent & \\
\hline \multicolumn{4}{|l|}{ Independent variable } \\
\hline Educational attainment (years) $(6=0)$ & $0-15$ & 6.45 & 2.85 \\
\hline \multicolumn{4}{|l|}{ Mediating variables } \\
\hline Interethnic contact & $0 / 1$ & 31.65 per cent & \\
\hline Associational involvement (\# org.) & $0-3$ & 1.03 & 1.11 \\
\hline Social trust & $0-4$ & 2.48 & 0.85 \\
\hline Euroscepticism & $0-4$ & 1.52 & 0.74 \\
\hline Perceived ethnic threat & $0-4$ & 1.82 & 0.75 \\
\hline Anti-Muslim attitudes & $0-4$ & 2.44 & 0.69 \\
\hline Nationalistic attitudes & $0-4$ & 2.82 & 0.79 \\
\hline Authoritarian attitudes & $0-4$ & 2.48 & 1.12 \\
\hline Present relative deprivation & $0-4$ & 1.24 & 0.84 \\
\hline Future economic decline & $0-4$ & 2.16 & 0.99 \\
\hline Political trust & $0-10$ & 5.63 & 1.58 \\
\hline \multicolumn{4}{|l|}{ Controls } \\
\hline Age $(18=0)$ & $0-52$ & 28.12 & 13.10 \\
\hline \multicolumn{4}{|l|}{ Gender } \\
\hline Male (ref.) & $0 / 1$ & 43.93 per cent & \\
\hline Female & $0 / 1$ & 56.07 per cent & \\
\hline Religiosity—church att. never/hardly ever (ref.) & $0 / 1$ & 56.07 per cent & \\
\hline Religiosity—church att. sometimes & $0 / 1$ & 26.10 per cent & \\
\hline Religiosity—church att. once a month or more & $0 / 1$ & 17.83 per cent & \\
\hline Marital status—not married (ref.) & $0 / 1$ & 29.20 per cent & \\
\hline Marital status-married & $0 / 1$ & 57.49 per cent & \\
\hline Marital status_-divorced/widowed & $0 / 1$ & 13.31 per cent & \\
\hline Occupational status high (ref.) & $0 / 1$ & 18.73 per cent & \\
\hline Occupational status low & $0 / 1$ & 14.34 per cent & \\
\hline Occupational status mid & $0 / 1$ & 37.34 per cent & \\
\hline Occupational status other (unemployed/students etc.) & $0 / 1$ & 29.59 per cent & \\
\hline Region north (ref.) & $0 / 1$ & 24.29 per cent & \\
\hline Region large cities & $0 / 1$ & 5.30 per cent & \\
\hline Region west & $0 / 1$ & 25.06 per cent & \\
\hline Region east & $0 / 1$ & 15.25 per cent & \\
\hline Region south & $0 / 1$ & 27.00 per cent & \\
\hline Region suburban district & $0 / 1$ & 3.10 per cent & \\
\hline
\end{tabular}

Source: Religion in Dutch Society (SOCON, 2011/2012) 